\title{
La sala de clases sí importa: incidencia del clima de aula sobre la percepción de intimidación y victimización entre escolares*
}

Classroom matters: The incidence of classroom climate on
perceptions of peer intimidation and victimization

Recibido: noviembre 20 de 2010 | Revisado: diciembre 12 de 2010 | Aceptado: enero 11 de 2011

\author{
VERÓNICA LÓPEZ** \\ MARÍA DE LOS ÁNGELES BILBAO \\ JosÉ IGNACIO RODRÍGUEZ \\ Pontificia Universidad Católica de Valparaíso, Chile
}

SICI: 2011-2777(201203)11:1<91:LSDCSI>2.0.CO;2-X

Para citar este artículo: López, V., Bilbao, M., Rodriguez, J. I. (2012). La sala de clases sí importa: incidencia del clima de aula sobre la percepción de intimidación y victimización entre escolares. Universitas Psychologica, 11(1), 91-101.

* Este estudio fue financiado por el proyecto FON. DECYT 11080055 y por PIA-CONICYT Proyecto CIE-05

** Escuela de Psicología, Av. El Bosque 1290 (Viña del Mar), Chile. Tel: (5632) 2274363. E-mail: veronica. lopez@ucv.cl

\section{RES UMEN}

La investigación sobre agresión entre escolares (bullying) tiende a poner énfasis en las características individuales de agresores y víctimas, y no así en las características contextuales. En este artículo presentamos evidencia de que el clima de aula es un factor predictor de la percepción de agresión entre escolares. Se aplicaron instrumentos de autopercepción y heteropercepción de intimidación y victimización de 444 estudiantes chilenos de séptimo y octavo básico, y se analizó el efecto de la percepción del clima de su sala de clases sobre estos indicadores. Para intimidación, el modelo de regresión fue significativo aún después de controlar el nivel socioeconómico y explicó el $40.1 \%$ de la varianza y para victimización, el $33.5 \%$. Específicamente, fueron las dimensiones de fricción, satisfacción y competencia las que incidieron en la intimidación y victimización entre pares. Estos resultados son discutidos en el marco de la investigación sobre victimización entre pares desde una perspectiva social-ecológica.

Palabras clave autor:

Bullying, violencia escolar, agresión entre escolares, clima de aula.

Palabras clave descriptores:

Perspectiva social-ecológica, educación media, desarrollo.

\section{A B S T R A C T}

Research on peer aggression (bullying) places emphasis on individual characteristics of aggressors and victims, and scarcely on context variables. This article provides evidence of classroom climate acting as predictor of perceived peer aggression. 444 students enrolled in sixth, seventh, and eight grade were administered instruments measuring self-reported and peer-reported intimidation and victimization. We analyzed the effect of classroom climate on these indicators. For intimidation, the regression model proved significant even after controlling for socioeconomic status, and explained $40.1 \%$ of variance; and for victimization, $33.5 \%$ of variance. Specifically, the dimensions of classroom climate associated with peer aggression were friction, satisfaction, and competitiveness. These results are discussed within the framework of research on peer aggression from a social-ecological perspective.

Key words author:

Bullying, school violence, peer aggression, classroom climate.

Key words plus:

Social-ecological perspective, secundary education, development. 


\section{Violencia escolar y bullying}

Las situaciones de violencia en contextos escolares han tomado gran notoriedad en la última década, siendo objeto tanto de estudio como de desarrollo de políticas públicas e intervenciones prioritarias (Abramovay, 2005; Gázquez, Pérez-Fuentes, Carrión \& Santiuste, 2010). La relevancia que tiene la convivencia y la violencia en la educación a nivel mundial, se ve reflejada en la declaración de la Unesco (2007) del período 2001-2010 como la "Década Internacional por una Cultura de Paz y No-Violencia para los Niños del Mundo". Entender mejor los elementos clave que participan de este fenómeno será, por lo tanto, fundamental para un abordaje exitoso de una construcción positiva de la convivencia escolar.

En Chile, las investigaciones iniciadas durante la segunda mitad de la década del 90 recogen la percepción de vulnerabilidad de las escuelas, especialmente de aquellas provenientes de contextos deprivados, frente a situaciones de violencia escolar. El estudio sobre convivencia escolar del año 2005 solicitado por el Ministerio de Educación y la Unesco (Instituto de Evaluación y Asesoramiento Educativo [Idea], 2005) reveló que el $86.5 \%$ de los estudiantes y el 91.7 \% de los docentes referían agresión en la convivencia escolar de sus establecimientos. En líneas similares, los resultados de la Encuesta Nacional de Violencia en el Ámbito Escolar, solicitado por los Ministerios de Educación e Interior a la Universidad Alberto Hurtado (Ministerio de Educación [Mineduc]/Ministerio del Interior [Minint], 2006), indicaron que el $96 \%$ de los alumnos y docentes percibían agresiones psicológicas en el establecimiento educacional y que el $83 \%$ de los alumnos (y el $61 \%$ de los profesores) percibía agresiones físicas en el colegio.

Estos estudios hablan de una alta percepción de violencia en el ámbito escolar. En ellos, la violencia escolar era entendida y analizada en términos genéricos, no observándose una desagregación por tipos o niveles de violencia. Así, actos de vandalismo eran agrupados en un nivel de análisis similar a las situaciones persistentes de agresión o intimidación entre pares. Los últimos esfuerzos en el país se han concentrado en dotar las evaluaciones de medidas más precisas y diferenciadas de agresión. Así, los resultados del último Estudio Nacional Violencia en el Ámbito Escolar (2009) indican que el $10.7 \%$ de los estudiantes son víctimas de bullying, es decir, sufren de un nivel de victimización persistente por parte de sus pares. Niveles similares de victimización han sido reportados por el último estudio de Fundación Paz Ciudadana (Varela, Farren \& Tijmes, 2010) y por López, Morales y Ayala (2009).

Por otra parte, los resultados de las primeras encuestas nacionales señalaban una violencia escolar entre escolares que estudian en establecimientos públicos municipalizados. Por ejemplo, los resultados de la Encuesta Nacional realizada el año 2005 (Mineduc/Minint, 2006) encontró que los estudiantes de nivel socioeconómico (NSE) bajo y dependencia municipal percibían mayor frecuencia de agresiones. En Chile, dada la alta segmentación social, que se corresponde de manera directa con la estratificación educativa de nuestro sistema educativo ${ }^{1}$, estos resultados tienden a reforzar la creencia popular de que la violencia está asociada a la pobreza.

Sin embargo, López et al. (2009) y López y Orpinas (2011), al estudiar la prevalencia y características de la agresión entre escolares en $6^{\circ}, 7^{\circ} \stackrel{\circ}{y}$ 8o año básico, no encontraron diferencias entre estudiantes de escuelas municipales, particularsubvencionadas y privadas en el nivel de agresión reportada, pero sí en el nivel de victimización percibida, donde los estudiantes de NSE medio-bajo, matriculados en escuelas municipales, percibían mayor victimización y se autopercibían más víctimas que los estudiantes de colegios privados. La aparente contradicción de estos resultados da cuenta de la

1 En Chile, los estudiantes provenientes de contextos de nivel socioeconómico bajo y medio-bajo estudian en su mayoría en escuelas públicas de administración municipal, mientras que aquellos de NSE medio-alto y alto están matriculados en colegios particulares de administración privada. Los estudiantes de NSE medio y medio-alto están matriculados en escuelas subvencionadas por el Estado, pero que son de administración privada y se denominan escuelas particular-subvencionadas. Durante la última década, se ha observado un aumento de la matrícula en escuelas particular-subvencionadas, en detrimento de las escuelas municipales y de los colegios privados. 
complejidad del fenómeno de estudio. Al parecer, la agresión entre escolares difiere según se midan percepciones y conductas de agresión, que según se midan niveles de victimización. Así también, no es lo mismo medir la agresión desde la percepción que uno tiene de sí mismo (autopercepción) que desde la percepción que mis compañeros de curso tienen de mí (heteropercepción) (López \& Orpinas, 2011). Por otra parte, estos resultados podrían explicarse si se toman en cuenta las características del contexto social y escolar asociadas a la vulnerabilidad, particularmente la estigmatización social ligada a una fragmentación social y educativa cada vez más notoria.

Como fenómeno complejo, la violencia escolar necesita ser analizada tomando con consideración la interrelación entre los distintos niveles participantes que operan en y a través de ella. En este sentido, el modelo ecológico de Bronfenbrenner (1989) resulta de interés, habiendo sido utilizado ya en la investigación de violencia escolar (Espelage \& Swearer, 2010; Khoury-Kassabri, Astor \& Benbenishty, 2009). Desde este modelo, el fenómeno de la violencia escolar se entiende inserto dentro de dinámicas relacionales de la escuela, que guardan relación con el clima de los espacios de aprendizaje y convivencia, así como con aspectos del entorno y de políticas del establecimiento educacional. Estas son las llamadas variables escolares, que inciden en mayor o menor grado en los niveles de intimidación y victimización (bullying), dependiendo del contexto que las rodee.

Este tipo de mirada difiere de la perspectiva tradicional en la investigación de corriente principal de bullying, que ha puesto énfasis particularmente en las características individuales de agresores, víctimas y espectadores. El propio Olweus (1993, 1998) adoptó el término bullying para denominar la figura de "bully" o 'matón', como un sujeto de mayor poder físico o social en relación con sus pares y quien, con la intención de hacer daño, produce una situación constante y persistente de victimización de un niño o grupo de niños (Olweus, 1998). Desde sus inicios, han proliferado los estudios y grupos de investigación dedicados a comprender las características personales de la llamada "tríada de bullying", características relacionadas con rasgos de personalidad, diferencias físicas y sociales, entre otras (Ortega, 1998; Smith, 2004).

Esta mirada adolece de una serie de limitaciones que significan verdaderas dificultades de cara a la intervención psicoeducativa. Si se sitúa el foco del problema en las características personales, entonces la intervención, a su vez, se centra en dichas características y en su modificación. Pero, dado que muchas de ellas son condiciones poco modificables (estatura, peso corporal, introversión/extroversión) o ambientalmente dependientes (estatus social), entonces las posibilidades de cambio son limitadas. Quizás por eso, han proliferado los modelos antibullying focalizados en el desarrollo de habilidades sociales, bajo el supuesto de que estas sí son educables y pueden producir cambios conductuales.

Una perspectiva de análisis que intenta superar el programa individualizador de la agresión entre pares, proviene de la Psicología Social de grupos. Aquí, los investigadores buscan comprender el bullying como un fenómeno grupal e intergrupal. Desde esta mirada, resulta importante destacar el rol participativo que ocupan los espectadores o testigos de la agresión, y las motivaciones de afiliación, pertenencia, estatus y poder que subyacen a las agresiones entre escolares (Berger \& Lisboa, 2009; Betancourt, 1997). Aún cuando esta perspectiva es necesaria, sigue situando el problema en los estudiantes y, por tanto, focaliza la acción en el grupo de pares, dejando de lado la participación de los adultos de la escuela (profesores, directivos, apoderados) en la construcción de ambientes de aprendizaje y de convivencia que propician la violencia escolar y la agresión entre estudiantes.

Desde la perspectiva social-ecológica (Swearer \& Espelage, 2004), la violencia escolar es un fenómeno en el cual se conjugan elementos de múltiples niveles de análisis; entre ellos, los niveles individuales, diádicos, grupales, sociales y culturales. Como señalan Espelage y Swearer (2010), "el fenómeno del bullying y de la victimización no ocurren de manera aislada y típicamente no ocurren entre un "matón" y una "víctima". De hecho, estos fenómenos corresponden a complejos intercambios sociales entre 
individuos, grupos de pares, y su ambiente social más amplio" (p. 61, traducción nuestra).

Como vemos, esta perspectiva no niega la influencia de variables de tipo individual y grupal, sino que las sitúa de manera contextualizada e inmersas dentro de fuerzas contextuales y culturales que dinamizan su efecto sobre las conductas de intimidación y victimización. Como señalan Berger y Lisboa (2009), "el análisis del comportamiento social, según este modelo teórico, remite a un nivel que integra tanto los comportamientos individuales, la dinámica de las interacciones sociales, como la formación de grupos, circunscritos en un contexto socio-cultural" (p. 62).

Así, comprender la violencia escolar implica también comprender la agresividad que ocurre a nivel de pares, es decir entre compañeros de curso, pero no se limita a ella, debiendo estudiarse, además, elementos del espacio escolar como constitutivo de relaciones, así como el sistema educativo en contextos socioculturales demarcados por políticas educativas particulares (Berger \& Lisboa, 2009; Debarbieux, 1997; Espelage \& Swearer, 2010; Khoury-Kassabri, Astor \& Benbenishty, 2009). Así, y desde la línea de investigación de la agresión entre pares, es necesario abordar su estudio teniendo en consideración la interrelación de los distintos niveles de análisis.

\section{Elementos explicativos de la agresión entre escolares: del individuo a la interacción en el aula}

Incluso desde la investigación clásica en bullying, la metáfora del triángulo implica una redefinición del concepto, desde una visión diádica a una triádica, que incorpora el rol activo de los observadores, como una audiencia que admite y promueve la victimización (Twemlow, Fonagy \& Sacco, 2010). Esto tiene importantes consecuencias para la intervención, puesto que el foco ya no estaría puesto en los individuos "diana" (target), agresores y víctimas, sino en el clima social y emocional del aula.

Los investigadores interesados en las dimensiones sociales del fenómeno de bullying y victimización entre escolares, han puesto énfasis en el papel socioemocional que juega el clima de aula (Assael
\& Neumann, 1991; Khoury-Kassabri, Benbenishty \& Astor, 2005; Yoneyama \& Rigby, 2006).

Por clima de aula entendemos la percepción de los alumnos y el profesor respecto de los aspectos del aula que influyen en la calidad de las relaciones y los aprendizajes (Villa \& Villar, 1992). Así, al interior de un establecimiento educacional puede coexistir una multiplicidad de climas de aula (Villa \& Villar, 1992). El desafío es generar investigación que muestre, con criterios de rigor científico, los efectos de estos elementos contextuales y situados en el escenario escolar.

En Chile, Arón y Milicic (1999, véanse también Milicic \& Arón, 2000) identificaron elementos que inhiben el desarrollo socioemocional de los estudiantes y, a su vez, favorecen la violencia escolar. Estos elementos, derivados fundamentalmente de sus estudios cualitativos, fueron: una concepción autoritaria de la educación, una rigidez excesiva en el concepto de jerarquía, sistemas de control coercitivos, una concepción unidireccional de las relaciones de respeto (tú me respetas a mí), una concepción de la obediencia que no permite la divergencia ("porque sí"), y la evitación del conflicto. Mena y Vizcarra (2001) plantearon que estos criterios definirían a los sistemas escolares maltratadores y maltratados. Toledo, Magendzo y Gutiérrez (2009) estudiaron la relación entre intimidación entre escolares, clima de aula y rendimiento escolar. Encontraron una relación significativa y negativa entre intimidación y clima de aula (a mayor frecuencia de intimidación, peor sentimiento de sentirse respetado en el curso) en estudiantes tanto de básica $(r=-0.25)$ como de media $(r=-0.27)$.

Los resultados de estudios muestran que es posible estudiar esta variable "intangible" de clima en la escuela, y que cuando se realiza con rigor, los resultados sugieren que sí hay relaciones, y que la fuerza de la relación es suficiente como para ser estadísticamente significativa. Como han señalado Cohen, McCabe, Michelli y Pickeral (2009), la investigación en clima escolar tiene una data de más de cien años en los países anglosajones, pero existe una brecha profunda entre el nivel de desarrollo de la investigación sobre clima escolar y el desarrollo de políticas públicas y de programas de prevención 
de violencia escolar que aborden los efectos del clima escolar.

Ahora bien, es necesario diferenciar, tanto conceptual como operativamente, el clima escolar del clima de aula. Assael y Neumann (1991) definen el clima escolar (CE) como un constructo multidimensional que hace referencia a las percepciones, pensamientos y valores que los miembros de un establecimiento educacional construyen de este y de las relaciones que en él se dan. El clima de aula, en cambio, refiere a la percepción de estudiantes y profesores respecto de los aspectos del aula que influyen en la calidad de las relaciones y los aprendizajes (Villa \& Villar, 1992).

Esta búsqueda por diferenciar clima escolar de clima de aula se aprecia en el estudio reportado por Espinoza (2006), quien estudió la relación entre el maltrato escolar y el rendimiento académico de estudiantes de Guatemala de escuelas públicas y privadas. Esta autora encontró una relación significativa y positiva entre la violencia entre iguales y el maltrato de los profesores a los estudiantes $(r=0.56)$. La magnitud de esta relación nos pone en alerta respecto del papel de los adultos en la intimidación entre pares. Por otra parte, el clima de aula y el clima escolar también presentaron una relación significativa y positiva con la violencia entre iguales, y negativa con el rendimiento escolar.

Considerando la necesidad de contar con mayor evidencia empírica respecto del papel del clima de aula sobre el fenómeno de bullying o victimización entre pares, el objetivo del presente artículo fue analizar la incidencia del clima de aula en la percepción y conductas de intimidación y victimización entre estudiantes en edad escolar. La hipótesis fue que el clima en el aula positivo disminuiría las conductas de intimidación y victimización dentro de los establecimientos educacionales.

\section{Metodología}

\section{Participantes}

Participaron 444 estudiantes de $7^{\circ}$ y $8^{\text {o }}$ básico, de establecimientos urbanos de las comunas de Viña del Mar y Valparaíso, Región de Valparaíso, de depen- dencia pública, privada y mixta. El diseño muestral fue intencionado y estratificado según curso escolar.

\section{Medidas e instrumentos}

a) Heteroinforme de intimidación y victimización: se utilizó la versión Heteroinforme-Pares del Insebull. Instrumentos para la evaluación de bullying (Avilés \& Elices, 2007). Este instrumento mide conductas de acoso escolar como agresión y victimización en estudiantes. El rango de respuestas es de 1 a 9. El instrumento cumple con propiedades psicométricas de confiabilidad (alpha entre 0.83 y 0.97 para las distintas formas) y validez (de contenido, mediante el juicio de expertos; y de constructo, mediante análisis factorial confirmatorio, que identificó dos factores principales: intimidación y victimización); b) Autoinforme de intimidación y victimización: se utilizaron las Escalas de Agresión y Victimización (Orpinas \& Frankowski, 2001) en su versión adaptada al español (López \& Orpinas, 2011). Constan de 21 ítems que evalúan el comportamiento del alumno en la última semana, cuántas veces ha sido victimizado y cuántas veces ha intimidado. El instrumento cumple con criterios de consistencia interna (alfa $=0.86$ y 0.85 ) y validez estructural (análisis factorial exploratorio y confirmatorio); c) Clima de aula: se utilizó el Inventario "Mi Clase" (Anderson, 1973 en Villa \& Villar, 1992), compuesto de 32 ítems que evalúan el clima de aula según cinco dimensiones: Satisfacción, Cohesividad, Fricción, Dificultad y Competitividad. Se empleó la versión adaptada semánticamente al contexto chileno por Ascorra y Cáceres (2002). La confiabilidad del instrumento en nuestro estudio, tras estudiar su estructura y eliminar por un mal funcionamiento algunos ítems y la dimensión Dificultad, fue adecuada con un alpha de Cronbach para la escala total de 0.77 (19 ítems) y en cada dimensión: Satisfacción 0.63 (6 ítems), Cohesividad 0.63 (6 ítems), Fricción 0.76 (3 ítems) y Competitividad 0.6 (4 ítems). Se realizó un análisis factorial exploratorio el cual confirmó la pertinencia de los ítems a cada dimensión y con el cual se construyeron las cuatro dimensiones utilizadas posteriormente. El análisis del Inventario Clima de Aula mostró una correlación alta entre las 
cuatro dimensiones del Inventario Mi Clase ( $r$ entre 0.86 -fricción y cohesividad-, y 0.93 -satisfacción y cohesividad-, todas significativas al nivel $p<0.001$ ), mostrando una alta consistencia interna.

\section{Procedimiento}

Una vez establecido el contacto con los establecimientos que participan en el estudio, los padres firmaron carta de consentimiento informado y los estudiantes, de asentimiento informado. Los instrumentos se aplicaron en los establecimientos durante el horario escolar. Los datos fueron analizados mediante el paquete estadístico SPSS versión 15.

\section{Resultados}

Relaciones entre Clima de Aula y los indicadores de Intimidación y Victimización

La Tabla 1 muestra los resultados del análisis correlacional entre los indicadores de Intimidación y Victimización utilizados en la investigación y las dimensiones del Clima de Aula. Las medidas de percepción de agresión, tanto desde el heteroinforme como desde el autoinforme, presentan correlaciones estadísticamente significativas con las dimensiones de Satisfacción, Fricción y Competitividad del Clima de Aula.

Mientras que la medida de percepción de intimidación medida con heteroinforme (a lo largo del año) muestra correlaciones significativas con una menor satisfacción $(r=-0.13, p<0.05)$ y mayor nivel de fricción $(r=-0.24, p<0.01)$, estando relacionados con un peor clima de aula; por otro lado, la Escala de Agresión (conductas de intimidación en la última semana), presenta una relación significativa con una menor competitividad dentro del aula $(r=-0.11, p<0.05)$. Por otra parte, las medidas de percepción de victimización medida con heteroinforme y con autoinforme muestran el mismo patrón. Como se observa en la Tabla 1, la percepción de victimización con heteroinforme presenta una correlación negativa con satisfacción del clima de aula $(r=-0.21, p<0.01)$, mientras la percepción de victimización con autoinforme presenta una correlación significativa con menor competitividad en el aula $(r=-0.14, p<0.01)$.

Estos resultados muestran que existen relaciones significativas entre la percepción de clima de aula, medida por el Inventario Mi Clase, y las escalas que informan sobre percepción y conducta de intimidación y victimización, tanto de la última semana como la percepción del último año. Con el fin de estudiar la influencia conjunta de las distintas dimensiones de clima de aula en la percepción de victimización y de intimidación, se realizaron regresiones lineales para las medidas de estas últimas.

\section{Regresión de clima de aula en medidas de percepción de intimidación}

Si bien la varianza de la percepción de intimidación, medida por heteroinforme, explicada por las dimensiones del clima de aula es baja $\left(R^{2}=0.07\right)$, el modelo es significativo $\left(F_{(4,363)}=6.515, p<0.001\right)$. Desde el heteroinforme, al haber mayores índices de Fricción $(B=-1.20, E T=0.299, \beta=-0.223$, $p<0.001$ ) habría mayores niveles de intimidación dentro del aula de clases. La influencia de la percepción de fricción se mantiene, aún controlando tanto las medidas de conducta de intimidación como las percepciones de victimización, como se observa en

TABLA 1

Correlación bivariada entre las dimensiones del Inventario Mi Clase y los índices de Intimidación y Victimización

\begin{tabular}{lcccc}
\hline \multicolumn{1}{c}{ Variable } & Satisfacción & Cohesividad & Fricción & Competitividad \\
\hline Heteroinforme-intimidación & $-0.128(*)$ & 0.071 & $-0.235(* *)$ & 0.041 \\
Heteroinforme-victimización & $-0.213(* *)$ & 0.097 & -0.077 & 0.076 \\
Escala de Agresión & -0.01 & -0.038 & -0.017 & $-0.105(*)$ \\
Escala de Victimización & -0.024 & -0.03 & -0.021 & $-0.136(* *)$ \\
\hline
\end{tabular}

Nota. $* p<0.05$; * $p<0.01$ (bilateral).

Fuente: elaboración propia. 
la Tabla 2 (fricción $B=-0.99, p<0.001$ ). El modelo con estas variables explica el $39.2 \%$ de la varianza $\left(\right.$ Cambio $\left.R^{2}=0.33 ; F_{(7,360)}=33.212, p<0.001\right)$. Dados los estudios que han informado que el nivel socioeconómico resultaría significativo en diferenciar distintos niveles de intimidación (véanse Antecedentes), se realizó una segunda regresión incorporando esta variable al modelo. El modelo resultó significativo, aunque el aporte de esta variable es ínfimo, explicando el 40,1\% (Cambio $R^{2}=0.01$, $\left.F_{(1,357)}=5.328, p<0.05\right)$ y sin alterar de manera significativa la influencia de las demás variables.
Por otro lado, al analizar la conducta de intimidación según autoinforme en relación con la influencia de las dimensiones de clima de aula, el modelo de regresión no resultó significativo $\left(F_{(4,363)}=1.545, p=0.189\right)$.

\section{Regresión de clima de aula en medidas de victimización}

La regresión de las dimensiones de Clima de Aula en la percepción de victimización con heteroinforme resultó significativa, con una varianza explicada

\section{TABLA 2}

Contribución de las dimensiones de clima de aula a la intimidación desde el heteroinforme

\begin{tabular}{|c|c|c|c|}
\hline Variable & $\mathrm{B}$ & ET & $\beta$ \\
\hline Satisfacción & 0.099 & 0.314 & 0.015 \\
\hline Cohesión & 0.297 & 0.344 & 0.038 \\
\hline Fricción & -0.985 & 0.243 & $-0.183(* *)$ \\
\hline Competitividad & 0.140 & 0.297 & 0.020 \\
\hline Hetero-Victimización & 0.938 & 0.071 & $0.565(* * *)$ \\
\hline Escala de Agresión & 0.068 & 0.024 & $0.141(* *)$ \\
\hline Escala de Victimización & -0.031 & 0.025 & -0.063 \\
\hline
\end{tabular}

Nota 1. $R^{2}=0.392 . * p<0.05 ; * * p<0.01 ; * * * p<0.001$

Nota 2. Variables predictoras: (Constante), dimensión de satisfacción, dimensión de cohesividad, dimensión de fricción, dimensión de competitividad, dimensión de competitividad, heteroinforme de intimidación, escala de agresión y escala de victimización. Variable dependiente: Heteroinforme Índice de Intimidación.

Fuente: elaboración propia.

TABLA 3

Contribución de las dimensiones de clima de aula a la victimización desde el heteroinforme

\begin{tabular}{|c|c|c|c|}
\hline Variable & B & ET & $\beta$ \\
\hline Satisfacción & -0.574 & 0.189 & $-0.140(* *)$ \\
\hline Cohesión & 0.206 & 0.210 & 0.044 \\
\hline Fricción & 0.195 & 0.151 & 0.060 \\
\hline Competitividad & 0.229 & 0.181 & 0.055 \\
\hline Hetero-Intimidación & 0.349 & 0.026 & $0.580(* * *)$ \\
\hline Escala de Agresión & -0.009 & 0.015 & -0.031 \\
\hline Escala de Victimización & 0.025 & 0.015 & 0.084 \\
\hline
\end{tabular}

Nota 1. $R^{2}=0.392$. $* p<0.05 ; * p<0.01 ; * * *<0.001$.

Nota 2. Variables predictoras: (Constante), dimensión de satisfacción, dimensión de cohesividad, dimensión de fricción, dimensión de competitividad, dimensión de competitividad, heteroinforme de intimidación, escala de agresión y escala de victimización. Variable dependiente: Heteroinforme Índice de Victimización.

Fuente: elaboración propia. 
pequeña de $R^{2}=0.05\left(F_{(4,363)}=5.227, p<0.001\right)$. Menores niveles de Satisfacción con el clima de aula determinan mayores niveles de victimización en Heteroinforme $(B=-0.811, \mathrm{ET}=0.231$, $\beta=-0.198, p<0.001)$. Esto se mantiene aun incorporando a las medidas otras medidas de victimización e intimidación, como se observa en la Tabla 3. El nivel socioeconómico no fue significativo para las medidas de victimización.

Por otro lado, al analizar la conducta de victimización desde el autoinforme en relación con la influencia de las dimensiones de clima de aula, el modelo de regresión resultó significativo $\left(F_{(4,363)}=2.708, p<0.05\right)$, aunque explica un bajo porcentaje de la varianza de $3 \%$ (véase Tabla 5). La dimensión que explicaría mayores niveles de victimización es un menor nivel de Competitividad en el aula $(B=-2.19, \mathrm{ET}=0.75, \beta=-0.157$, $p<0.01)$. La influencia de la percepción de una baja competitividad se mantiene, aun controlando tanto las medidas de conducta de intimidación como las percepciones de victimización, como se observa en la Tabla 5 (Fricción $B=-1.28, p<0.05$ ). El modelo con estas variables explica el 33,5\% de la varianza $\left(\right.$ Cambio $\left.R^{2}=0.30 ; F_{(7,360)}=25.928, p<0.001\right)$.

\section{Conclusión}

Los resultados muestran que la percepción de intimidación de un estudiante, desde el punto de vista de los pares (heteroinforme) está influida por altos niveles de fricción en el aula que ese estudiante percibe. Sin embargo, las conductas de intimidación medidas según el autoinforme (el que mide frecuencia de conductas de agresión realizadas durante la última semana) no aparecen influidas de manera significativa por las dimensiones de clima de aula. Cabe señalar que el NSE del establecimiento resultó ser significativo en el caso de la percepción de intimidación, resultados que requieren de estudios más acabados a futuro. Sin embargo, tras controlar el efecto del NSE, el modelo se mantuvo significativo.

Por otro lado, la heteropercepción de victimización (es decir, el que los compañeros de curso perciban a un estudiante como victimizado) estaría influida por bajos niveles de satisfacción de ese estudiante con el clima de su aula. A su vez, la autopercepción de victimización (vale decir, el que un estudiante autorreporte alta frecuencia de victimización durante la última semana), estaría influido por una percepción de bajo nivel de competitividad en el aula. Este último resultado podría ser explicado por una falta de concentración en temáticas del proceso de aprendizaje, pero merece una atención más profunda en futuras investigaciones para aclarar su significado.

Estos resultados dan soporte a la hipótesis de que un clima de aula positivo disminuye de forma significativa las percepciones de intimidación en el último año, así como la percepción y las conductas

TABLA 5

Contribución de clima de aula a la victimización desde el autoinforme

\begin{tabular}{|c|c|c|c|}
\hline Variable & $\mathrm{B}$ & ET & $\beta$ \\
\hline Satisfacción & -0.011 & 0.668 & 0.001 \\
\hline Cohesión & 0.112 & 0.733 & 0.007 \\
\hline Fricción & -0.437 & 0.528 & -0.04 \\
\hline Competitividad & -1.283 & 0.628 & $-0.092(*)$ \\
\hline Hetero-Intimidación & -0.14 & 0.183 & 0.09 \\
\hline Hetero-Victimización & -0.303 & 0.112 & -0.069 \\
\hline Escala de Victimización & 0.543 & 0.043 & $0.555(* * *)$ \\
\hline
\end{tabular}

Nota 1. $R^{2}=0.392$. * $p<0.05 ; * *<<0.01 ; * * p<0.001$

Nota 2. Variables predictoras: (Constante), dimensión de satisfacción, dimensión de cohesividad, dimensión de fricción, dimensión de competitividad, heteroinforme de intimidación, heteroinforme de victimización y escala de victimización. Variable dependiente: Autoinforme Victimización.

Fuente: elaboración propia. 
de victimización. La heteropercepción de intimidación durante el año escolar se ve significativamente afectada por altos niveles de fricción en la clase, mientras que la autopercepción de intimidación durante la última semana se ve afectada por bajos niveles de competitividad. Por otra parte, una menor satisfacción con el curso explicaría mayores niveles de victimización (heteroinforme), y menores niveles de competitividad explicarían mayores niveles de victimización (autoinforme). Estos resultados sugieren que mientras más negativamente un estudiante perciba el ambiente de su sala de clases en términos de fricción y competitividad, es más probable que sus compañeros consideren que ese estudiante participa frecuentemente en acciones de intimidación; y que mientras menos satisfecho se encuentre un estudiante con el grupo curso y menos competitiva perciba la dinámica del curso, es más probable que sus compañeros lo perciban como altamente victimizado.

Estos resultados nos permiten proponer que, al aumentar o disminuir la satisfacción, la fricción y la competitividad, dimensiones del clima del aula, se producirán variaciones en las percepciones y las conductas de intimidación y victimización del curso. Esta conclusión es relevante de cara a la intervención psicoeducativa, pues pone el foco en las variables contextuales que influyen en la violencia escolar. La lógica es la siguiente: si encontramos una asociación entre los ambientes de aprendizaje de la sala de clases (clima de aula) y los niveles de agresión escolar, entonces podemos suponer que, actuando sobre los climas de aula, podemos disminuir los niveles de agresión escolar. En efecto, este estudio aporta evidencia a favor de dicha asociación. Aún cuando, como cualquier estudio de corte transversal, el estudio está limitado en sus posibilidades de probar relaciones de causalidad temporal, la asociación y las orientaciones para la acción propuestas teóricamente son coherentes con la perspectiva social-ecológica desde la cual se abordó esta investigación (Espelage \& Swearer, 2010).

Cabe destacar que es la heteropercepción de agresión (y no la autopercepción) la medida que más se ve afectada por las variables de clima de aula. Estos resultados sugieren que la percepción de bullying que tienen los estudiantes es, de manera importante, una construcción grupal de representaciones respecto de clima de aula. Esto podría explicar las diferencias reportadas en Chile respecto de la evaluación de la percepción de "violencia escolar", que suelen ser mayores que las medidas de "agresión entre escolares". Esto es muy importante, pues muestra que la intervención en bullying requiere de una acción colectiva, no solo sobre el grupo curso, sino también incluyendo al profesor. Como hemos señalado anteriormente, el profesor participa activamente en la construcción del climas "tóxicos" o "nutritivos" para el aprendizaje (Milicic \& Arón, 2000). En el caso de los establecimientos de este estudio, los resultados nos indican que al haber mejor clima de aula, habría menos conductas de intimidación y victimización. Por lo tanto, al haber un buen clima de aula, positivo, inclusivo, con poca fricción entre sus estudiantes y mayores niveles de satisfacción, habría menos bullying en la sala de clases. Estos resultados son concordantes con los resultados de Unesco (Blanco, 2005; Unesco, 2008) respecto del papel preponderante del clima escolar como elemento predictor del rendimiento académico pues, como señalan Blanco (2005) y Cohen (2006), la existencia de un buen clima afectivo y emocional en la escuela y en el aula, es una condición fundamental para que los alumnos aprendan y participen plenamente en la clase.

\section{Referencias}

Abramovay, M. (2005). Violencia en las escuelas, un gran desafío. Revista Electrónica Iberoamericana de educación, 38, 53-66.

Arón, A. \& Milicic, N. (1999). Clima social escolar y desarrollo personal. Un programa de mejoramiento. Santiago: Editorial Andrés Bello.

Ascorra, P. \& Cáceres, P. (2002). Evaluación de los aspectos psicométricos del Inventario de Clima de Aula "Mi Clase". Revista Enfoques Educacionales, 3(2), 117-134.

Assael, J. \& Neumann, E. (1991). Clima emocional del aula. Santiago: PIIE.

Avilés, J. M. \& Elices, J. A. (2007). Insebull. Instrumentos para la evaluación del bullying. Madrid: CEPE. 
Berger, C. \& Lisboa, C. (2009). Violencia escolar: estudios y posibilidades de intervención en Latinoamérica. Santiago de Chile: Editorial Universitaria.

Betancourt, H. (1997). An attribution model of social conflict and violence: From psychological to intergroup phenomena. Psykhe, 6(2), 3-12.

Blanco, R. (2005). Los docentes y el desarrollo de escuelas inclusivas. Revista Preal, 1, 174-177.

Bronfenbrenner, U. (1989). Ecological systems theory. Annals of Child Development, 6, 187-249.

Chile, Instituto de Evaluación y Asesoramiento Educativo. (2005). Primer estudio nacional de convivencia escolar: la opinión de docentes y estudiantes 2005: informe final [en línea]. Disponible en http:// www.unesco.cl/medios/biblioteca/documentos/ primer_estudio_nacional_convivencia_escolar_ informe_final_2005.pdf

Chile, Ministerio de Educación/Ministerio del Interior. (2006). Principales resultados del estudio nacional de violencia en el ámbito escolar. Santiago de Chile: Autores.

Chile, Ministerio de Educación. (2009). Segundo estudio nacional de violencia en el ámbito escolar. Santiago de Chile: Autor.

Cohen, J. (2006). Social, emotional, ethical and academic education: Creating a climate for learning, participation in democracy and well-being. Harvard Educational Review, 76(2), 201-237.

Cohen, J., McCabe, L., Michelli, N. M. \& Pickeral, T. (2009). School climate: Research, policy, teacher education and practice. Teachers College Record, 111(1), 180-213.

Debarbieux, E. (1997). La violencia en la escuela francesa: análisis de la situación, políticas públicas e investigaciones. Revista de Educación, 313, 79-93.

Espelage, D. L. \& Swearer, S. M. (Eds.). (2004). Bullying in American schools: A social-ecological perspective on prevention and intervention. Mahweh, NJ: Lawrence Erlbaum Associates.

Espelage, D. L. \& Swearer, S. M. (2010). A social-ecological model for bullying prevention and intervention: Understanding the impact of adults on the social ecology of youngsters. En S. R. Jimenson, S. S. Swearer \& D. L. Espelage (Eds.), Handbook of bullying in schools: An international perspective (pp. 61-86). Nueva York: Routledge.
Espinoza, E. (2006). Impacto del maltrato escolar en el rendimiento académico. Revista Electrónica de Investigación Psicoeducativa, 4(9), 221-238.

Gázquez, J.J., Pérez-Fuentes, M. C., Carrión, J. J. \& Santiuste, V. (2010). Estudio y análisis de conductas violentas en Educación Secundaria en España. Universitas Psychologica, 9(2), 371-380.

Khoury-Kassabri, M., Astor, R. A. \& Benbenishty, R. (2009). Middle Eastern adolescents' perpetration of school violence against peers and teachers: A cross-cultural and ecological analysis. Journal of Interpersonal Violence, 24(1), 159-182.

López, V., Morales, M. \& Ayala, A. (2009). Maltrato entre pares: conductas de intimidación y victimización en escolares chilenos. Revista de Psicología, 27(2), 243-286.

López, V. \& Orpinas, P. (2011). Las Escalas de Agresión y Victimización: prevalencia y características en estudiantes chilenos. Manuscrito enviado para publicación.

Mena, M. I. \& Vizcarra, R. (2001). Algo nuevo bajo el sol: hacer visible el maltrato y posible la esperanza. Psykhe, 10(1), 81-101.

Milicic, N. \& Arón, A. M. (2000). Climas sociales tóxicos y climas sociales nutritivos para el desarrollo personal en el contexto escolar. Psykhe, 9(2), 117-123.

Olweus, D. (1993). Bullying at school: What we know and what we can do. Oxford: Blackwell Publishing.

Olweus, D. (1998). Conductas de acoso y amenaza entre escolares. Madrid: Morata.

Orpinas, P. \& Frankowski, R. (2001). The Aggression Scale: A self-report measure of aggressive behavior for young adolescents. Journal of Early Adolescence, 21(1), 51-68.

Ortega, R. (1998). Víctimas, agresores y observadores. Alumnos implicados en situación de violencia. Madrid: Morata.

Smith, P. K. (2004). Bullying: Recent developments. Child and Adolescent Mental Health, 9, 98-103.

Toledo, M. I., Magendzo, A. \& Gutiérrez, V. (2009). Relación entre intimidación (bullying) y clima en la sala de clases y su influencia sobre el rendimiento de los estudiantes (Informe Final presentado al Fondo de Investigación y Desarrollo en Educación, FONIDE). Santiago: Ministerio de Educación. 
Twemlow, S., Fonagy, P. \& Sacco, F. (2010). The etiological cast to the role of the bystander in the social arquitecture of bullying and violence in schools and communities. En S. R. Jimerson, S. S. Swearer \& D. L. Espelage (Eds), Handbook of bullying in schools: An international perspective (pp. 73-86). Nueva York: Routledge.

UNESCO. (2007). Decenios y días internacionales de las Naciones Unidas. Disponible en http://www. unesco.org/education/es/sector

UNESCO. (2008). Los aprendizajes de los estudiantes de América Latina y el Caribe. (Primer reporte del Se- gundo Estudio Regional Comparativo y Educativo, SERCE). Santiago de Chile: Autor.

Yoneyama, S. \& Rigby, K. (2006). Bully/victim students \& classroom climate. Youth Studies Australia, 25(3), 34-41.

Varela, J., Farren, D. \& Tijmes, C. (2010). Violencia escolar en educación básica: evaluación de un instrumento para su medición. Santiago, Chile: Fundación Paz Ciudadana.

Villa, S. \& Villar, A. (1992). Clima organizacional y de aula: teorías, modelos e instrumentos de medida. San Sebastián: Servicio Central de Publicaciones del Gobierno Vasco. 
